\title{
Effect of statins on the treatment of early diabetic nephropathy: a systematic review and meta-analysis of nine randomized controlled trials
}

\author{
Jinyang $\mathbf{L v}^{1}$, Chunli Ren ${ }^{2}$, Qianpei Hu ${ }^{3}$ \\ ${ }^{1}$ Department of Anus-Intestines, Yongchuan Hospital Affiliated to Chongqing Medical University, Chongqing, China; ${ }^{2}$ Department of Respiratory \\ and Critical Care Medicine, Yongchuan Hospital Affiliated to Chongqing Medical University, Chongqing, China; ${ }^{3}$ Department of Nephropathy and \\ Rheumatism, Yongchuan Hospital Affiliated to Chongqing Medical University, Chongqing, China \\ Contributions: (I) Conception and design: J Lv; (II) Administrative support: C Ren; (III) Provision of study materials or patients: Q Hu; (IV) \\ Collection and assembly of data: All authors; (V) Data analysis and interpretation: All authors; (VI) Manuscript writing: All authors; (VII) Final \\ approval of manuscript: All authors. \\ Correspondence to: Qianpei Hu. Department of Nephropathy and Rheumatism, Yongchuan Hospital Affiliated to Chongqing Medical University, \\ Chongqing 402160, China. Email: a043488@163.com.
}

\begin{abstract}
Background Diabetic nephropathy (DN) is a secondary disease of diabetes and could cause serious renal damage. This article aimed to investigate the effect of statins on the treatment of early DN.

Methods: The databases were searched: Embase (January 2000-August 2021), PubMed (January 2000August 2021), Cochrane [randomized controlled trial (RCT) database], Ovid (January 2000-August 2021), and clinicaltrials.gov (January 2000-August 2021) to obtain RCT literature related to statin intervention and DN. After screening, the risk of bias assessment was performed using the RevMan 5.4 software bias assessment tool, which was then used to perform the meta-analysis and obtain the therapeutic effects of statins by estimating indicators such as estimated glomerular filtration rate (eGFR), serum creatinine (SCR), total cholesterol (TC) level, total triglyceride (TG), and high-sensitivity C-reactive protein (hs-CRP).

Results: A total of 9 articles, 3,426 patients, and 5 types of statins were included. Meta-analysis showed that after treatment, eGFR in the experimental group was higher than in the control group [mean difference $(M D)=5.80 ; 95 \%$ confidence interval $(C I):(2.21,9.40) ; \mathrm{P}=0.002]$, SCR was lower than in the control group $[\mathrm{MD}=-0.46 ; 95 \% \mathrm{CI}:(-0.69,-0.24)$; $\mathrm{P}<0.0001]$, hs-CRP level was lower than in the control group $[\mathrm{MD}=-1.20 ; 95 \% \mathrm{CI}:(-2.05,-0.36) ; \mathrm{P}=0.005]$, TC level was lower than in the control group $[\mathrm{MD}=-54.09$; 95\% CI: $(-68.02,-40.16) ; \mathrm{P}<0.00001]$, and TG level was lower than that in the control group $[\mathrm{MD}=-42.19$; 95\% CI: (-55.54, -28.84); $\mathrm{P}<0.00001]$.
\end{abstract}

Discussion: Statins can significantly increase eGFR, reduce SCR, decrease CRP level, and decrease blood lipid level in the treatment of DN, thus reducing the inflammatory response and protecting the kidney.

Keywords: Statins; diabetic nephropathy (DN); meta-analysis

Submitted Aug 30, 2021. Accepted for publication Oct 22, 2021.

doi: $10.21037 /$ apm-21-2673

View this article at: https://dx.doi.org/10.21037/apm-21-2673

\section{Introduction}

Diabetic nephropathy (DN) is a secondary disease of diabetes characterized by renal damage lasting more than 3 months and decreased estimated glomerular filtration rate (eGFR), the main pathological changes were mesangial cell proliferation and hypertrophy, the increase of extracellular matrix (ECM), and the progressive deterioration of renal function, which would lead to the development of endstage renal disease $(1,2)$. In the pathogenesis of $\mathrm{DN}$, lipid metabolism disorders are important risk factors, 
and hyperlipidemia-produced oxidized lipoproteins can exacerbate inflammation stimulation, leading to abnormal expression of fibrogenic cytokines in serum and inducing apoptosis, leading to apoptosis massive macrophage intervention, resulting in local tissue damage (3). Diabetes is closely related to hyperlipidemia. According to statistics, more than half of diabetic patients have dyslipidemia (4). Statins have obtained a lot of evidence-based medical evidence in the prevention and treatment of DN, and their main function is to reduce lipid synthesis by inhibiting hydroxymethylglutaryl coenzyme A reductase, thus reducing serum cholesterol content (5). Recent studies (6) have shown that statins can reduce cell injury and protect renal function by improving vascular endothelial function, inhibiting thrombosis, antioxidation, and regulating immunity. However, such studies have a small sample size and different study quality, which cannot provide sufficient evidence-based basis. In this study, a meta-analysis of existing randomized controlled studies was performed to provide stronger evidence. We present the following article in accordance with the PRISMA reporting checklist (available at https://dx.doi.org/10.21037/apm-21-2673).

\section{Methods}

\section{Study data}

Retrieve the clinical randomized controlled literature in English in the main medical databases. Inclusion criteria: (I) study type: the literature published after January 2000 was limited to randomized controlled trials (RCTs), and the language was English; (II) study subjects: all patients were diagnosed with diabetes and diagnosed with clinical stage 2 or $3 \mathrm{DN}$ (7), the urinary albumin output was $30-300 \mathrm{mg}$, and the age of the patients was not limited; (III) intervention methods: the observation group was treated with one of the statin therapies (pravastatin, simvastatin, lovastatin, atorvastatin, rosuvastatin, fluvastatin, pitavastatin), and was treated with a single drug, not together with other drugs. The control group was treated with a placebo only or with an angiotensin-converting enzyme inhibitor; (IV) outcome measures: including eGFR, serum creatinine (SCR), total cholesterol (TC) level, total triglyceride (TG), and highsensitivity C-reactive protein (hs-CRP). Exclusion criteria of literature: (I) patients with abnormal urine protein caused by other causes; (II) study on combined intervention with multiple drugs; (III) experiment with animals (including white mice) as study subjects; (IV) articles with incomplete or no observation indicators provided; (V) literature of review nature, case study, guideline, systematic analysis, and other non-RCT literature.

\section{Literature search strategy}

Retrieval database: Embase (January 2000-August 2021), PubMed (January 2000-August 2021), Cochrane (RCT database), Ovid (January 2000-August 2021), clinicaltrials. gov (January 2000-August 2021), search mode: keyword rapid search, input keywords: (Statins/pravastatin/ Simvastatin/lovastatin/Atorvastatin/Rosuvastatin/ Fluvastatin/Pitavastatin) AND (Diabetic nephropathy/ Chronic kidney disease/Diabetic kidney disease).

\section{Literature selection and data extraction}

The articles' screening was independently completed by two researchers, discussing and consulting to a third researcher in case of inconsistencies during this process. Excel was used to extract the data; if there was no data available for collection, the original author contacted them to fetch the data; if that did not work, the article was not included in the meta-synthesis. The two researchers cross-checked the data after collecting. The extracted data contents included: (I) basic data of literature: publication time, author and region; (II) characteristics of study subjects: patient age, sex ratio, duration of diabetes and HbA1c level; (III) literature intervention methods: intervention methods and observation time of two groups; (IV) outcome data: eGFR, SCR, TC level, TG and hs-CRP. In data extraction, if different types of literature use different units for the same indicator, data conversion shall be performed: the exchange formula of $\mathrm{mg} / \mathrm{dL}$ and $\mathrm{mmol} / \mathrm{L}$ is: $\mathrm{A}=18 \times \mathrm{B}$ (where $\mathrm{A}$ is the value in $\mathrm{mg} / \mathrm{dL}$ and $\mathrm{B}$ is the value in $\mathrm{mmol} / \mathrm{L}$ ).

\section{Statistical methods}

$\mathrm{I}^{2}$ test and $\mathrm{Q}$ test were used to analyze the heterogeneity between different studies. $\mathrm{I}^{2}<50 \%$ or $\mathrm{P} \geq 0.1$ indicated no statistical significance of heterogeneity among the literature. The variables eGFR, SCR, TC, TG, and hs-CRP counted in this study were all continuous variables, expressed as mean difference (MD), with $95 \%$ confidence interval (CI), and $\mathrm{P}<0.05$ considered statistically significant. For each outcome measure, all the literature reported each of the variables were included for final synthesis. If there was no statistical heterogeneity in the literature, the fixed-effects 
model was used. If there was heterogeneity, the random effect model was used; if less than 10 pieces of literature were included in the synthesis, publication bias was not performed. The analysis tool in this study used RevMan 5.4 software provided by Cochrane to present the analysis results in the form of a forest plot.

\section{Risk of bias and sensitivity analysis}

The risk of bias assessment was performed using the bias assessment tool integrated with RevMan 5.4. The risk assessment of each literature was performed from several aspects: randomization, allocation concealment, quality of blinding, outcome assessment, incomplete data, selective reporting, and other biases. For synthesis with statistical significance of heterogeneity, sensitivity analysis was performed, and if the source of heterogeneity could not be identified, general descriptive analysis was used.

\section{Results}

\section{Literature screening results}

In this search, a total of 78 articles were retrieved from Embase, 134 articles were retrieved from PubMed, 25 articles were retrieved from Cochrane search, 44 articles were retrieved from Ovid search, 10 articles were from clinicaltrails.gov, 291 articles were retrieved from related articles in total, all articles used the deduplication function of EndNote X9, 33 repeated articles (the remaining 258) were excluded, 198 articles that obviously did not meet the requirements were filtered out by reading the titles and abstracts (78 articles that did not meet the study subjects, 73 articles that did not meet the intervention methods, 45 articles that did not meet the control methods, 2 articles had no outcome measures), 60 articles were left, the full text of articles was obtained, and the articles were excluded after reading one by one. Finally, 9 articles with a total of 3,426 patients were included, as shown in Figure 1, and the basic characteristics of the articles are shown in Table 1.

\section{Risk assessment of bias of included articles}

RevMan 5.4 was used. The risk assessment of the included articles showed that except for the articles (12), which did not mention the randomization method, the other articles had a detailed description of the grouping method, the concealment of allocation, and the blind method. However, some articles did not mention the drop-out cases, which may cause attrition bias to the results, as shown in Figures 2,3.

\section{Meta-analysis results}

eGFR (mL/min/1.73 $\left.\mathrm{m}^{2}\right)$

Four articles $(8,9,11,16)$ reported the effect of different treatment methods on the eGFR of patients, including 3,067 patients. The number of patients in the experimental group and the control group was 1,543 and 1,524. Cause there was statistical heterogeneity between the articles $\left(\mathrm{I}^{2}=77 \% ; \mathrm{P}=0.004\right)$, the random effects model combined analysis was used, and the experimental group was superior to the control group for the improvement of eGFR [MD $=5.80 ; 95 \%$ CI: $(2.21,9.40) ; \mathrm{P}=0.002]$. As shown in Figure 4 .

\section{SCR (mg/dL)}

Five articles $(8,11,13-15)$ reported the effect of different treatment modalities on SCR in patients. Three hundred and eighty-seven patients were included, with 194 and 193 patients in the experimental and control groups. Cause there was statistical heterogeneity between the articles $\left(\mathrm{I}^{2}=97 \% ; \mathrm{P}<0.00001\right)$, random effects model combined analysis was used, and the improvement effect of the experimental group for SCR was better than the control group $[\mathrm{MD}=-0.46 ; 95 \% \mathrm{CI}:(-0.69,-0.24) ; \mathrm{P}<0.0001]$. The results are shown in Figure 5.

\section{hs-CRP (mg/L)}

Four articles $(8,11,12,15)$ reported the effect of different treatment methods on hs-CRP in patients. Three hundred and twenty-three patients were included, with 162 and 161 patients in the experimental and control groups. Cause there was statistical heterogeneity between the articles $\left(\mathrm{I}^{2}=93 \% ; \mathrm{P}<0.00001\right)$, random effects model combined analysis was used, and the experimental group was superior to the control group for the improvement of hs-CRP $[\mathrm{MD}=-1.20 ; 95 \% \mathrm{CI}:(-2.05,-0.36) ; \mathrm{P}=0.005]$. As shown in Figure 6.

\section{TC (mg/dL)}

Six pieces of literature $(8-11,14,15)$ reported the effect of different treatment methods on TC. A total of 392 patients were included. The number of patients in both the experimental group and the control group was 196. Cause there was statistical heterogeneity between the articles $\left(I^{2}=84 \% ; \mathrm{P}<0.00001\right)$, the random effects model combined analysis was used. The improvement effect of TC 

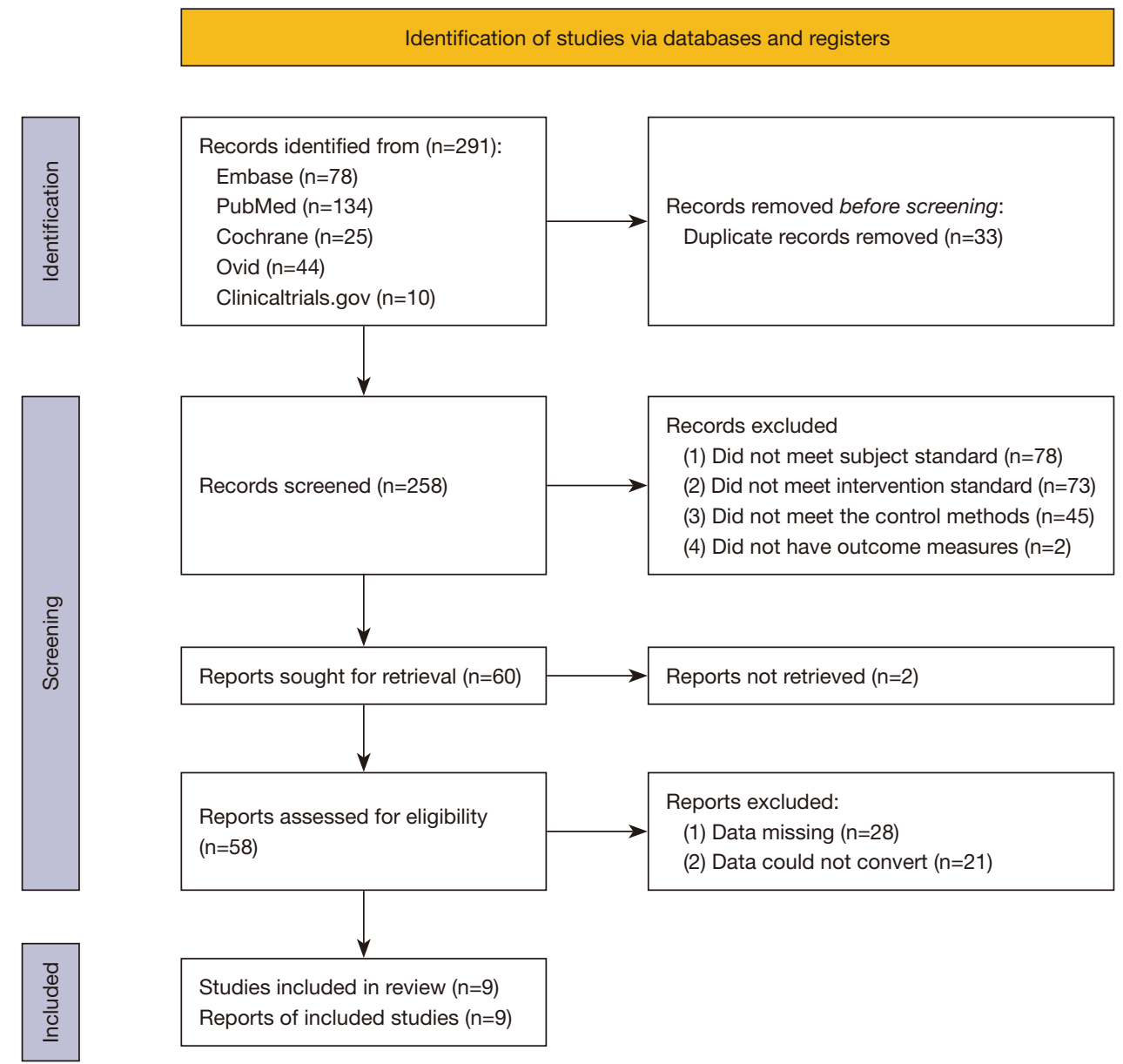

Figure 1 The search and selection flowchart.

in the experimental group was better than in the control group [MD $=-54.09$; 95\% CI: $(-68.02,-40.16) ; \mathrm{P}<0.00001]$. As shown in Figure 7.

\section{TG (mg/dL)}

Four literature $(8,10,12,15)$ reported the effect of different treatment methods on TG. A total of 323 patients were included. The number of patients in the experimental group and the control group was $161 / 162$. Since there was no statistical heterogeneity between the articles $\left(I^{2}=0 \%\right.$; $\mathrm{P}=0.83$ ), the combined analysis of the fixed-effect model was used. The experimental group was superior to the control group in the improvement effect of TG $[M D=-42.19 ; 95 \%$ CI: $(-55.54,-28.84) ; \mathrm{P}<0.00001]$. As shown in Figure 8.

\section{Publication bias analysis and sensitivity analysis}

From the results of the above comprehensive analysis, there was statistically significant heterogeneity among the articles. The source of heterogeneity may be the different characteristics of the patients and the intervention methods of the included studies, of which the important reason is to cause different statins used in the studies. The drugs involved in the eight articles included atorvastatin, cerivastatin, lovastatin, simvastatin, and pitavastatin. The different doses and methods of the drugs used and the different intervention methods in the control group caused considerable heterogeneity between the studies. Furthermore, because the number of articles included for each comprehensive analysis in the study was too small, no publication bias analysis was performed.

\section{Discussion}

Eight articles were included in this study, involving using 
Table 1 Basic characteristics table, object characteristics, intervention methods, and outcome measures of included articles

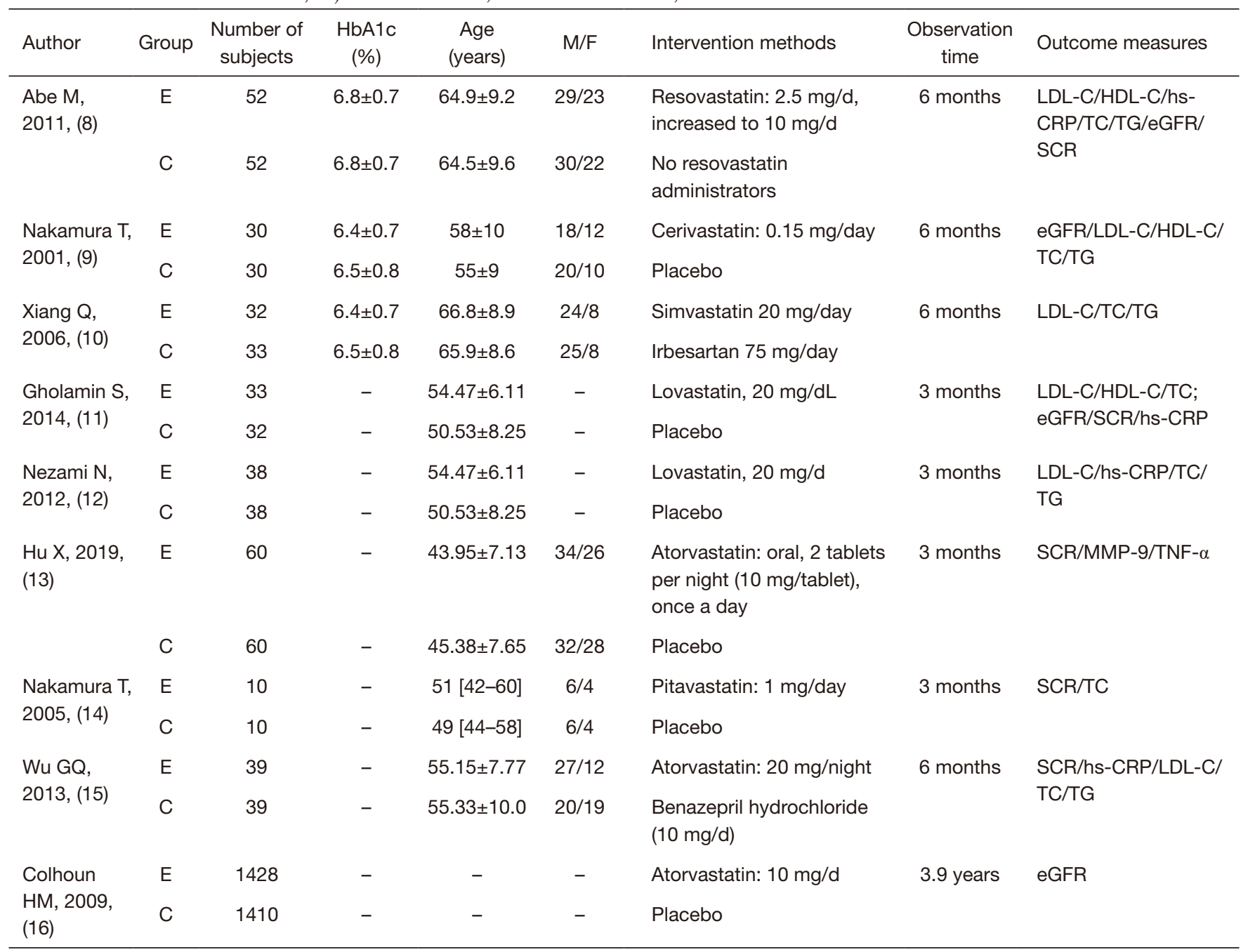

E, experimental group; C, control group; LDL-C, low-density lipoprotein cholesterol; HDL-C, high-density lipoprotein cholesterol; hs-CRP, high-sensitivity C-reactive protein; TC, total cholesterol; TG, total triglyceride; eGFR, estimated glomerular filtration rate; SCR, serum creatinine.

five statins (atorvastatin, cerivastatin, lovastatin, simvastatin, pitavastatin). The results of the final comprehensive analysis showed that statins had the following effects: (I) they could significantly increase the eGFR, reduce SCR, thus improving renal function; (II) they could reduce CRP levels, reduce the inflammatory response, thus protecting the kidney; (III) they could significantly reduce blood lipid levels and eliminate the root causes of DN.

The lipid-lowering effect of statins has been evidencebased, supported by a large number of clinical studies $(17,18)$, and this meta-analysis also provides strong evidence. Hyperlipidemia can affect local renal hemodynamics, promote glomerular sclerosis, and accelerate the occurrence of DN. Statins also dredge the local renal vessels while lowering lipid, reduce the deposition of lipids in the vascular wall, play the same role as angiotensin-converting enzyme inhibitors, thereby significantly reduce glomerular hypertension and high perfusion, alleviate the hyperfiltration of early DN, reduce urinary protein, improve hemodynamics, inhibit mesangial cell proliferation and ECM synthesis, and delay the progress of renal disease (19). A study (20) has indicated that statins can improve nitric oxide activity in the vascular endothelium, thus improving vascular endothelial function and relieving vascular endothelial contraction. Furthermore, diabetes belongs to immune diseases and is an inflammatory response mediated by a series of inflammatory 
factors, while statins can reduce the inflammatory response and stabilize platelet function (21).

However, it can be seen from the heterogeneity analysis of the literature in this study that there are significant pharmacodynamic differences between different statins.

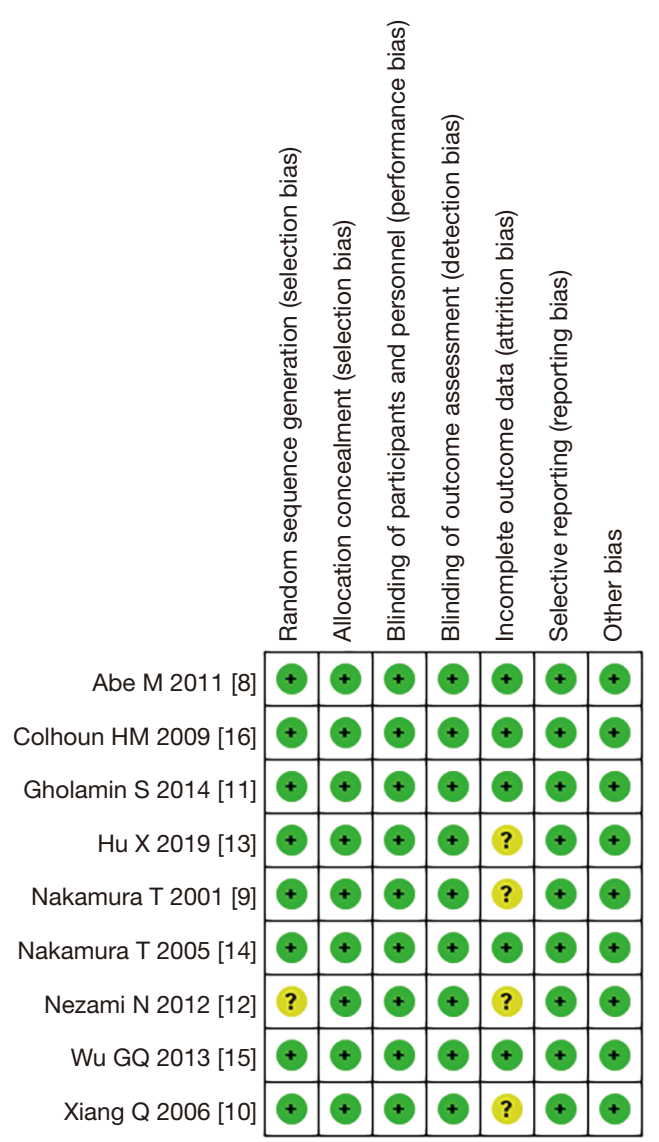

Figure 2 Summary chart of risk of bias assessment of included articles (8-16).
In our study, no comparative analysis was made on the lipid-lowering effect between drugs, but study pointed out that rosuvastatin and atorvastatin have an effective lipidlowering rate of $41 \%$, which are potent statins, while the lipid-lowering rate of other statins is about $30 \%$, which were conventional statins (22). A study by Vlad et al. (23) pointed out that atorvastatin has a good protective effect on the kidney when atorvastatin and rosuvastatin are applied to treat type $2 \mathrm{DN}$, and it acted safely. In the study by Kimura (24), pitavastatin was compared with pravastatin in treating patients with type $2 \mathrm{DN}$, and the results showed that pitavastatin was more effective in reducing urinary albumin. Studies (25) have shown that although highdose rosuvastatin reduces blood lipid concentrations to a greater extent than high-dose atorvastatin, atorvastatin seems to have a renoprotective effect in the chronic kidney disease population studied. Additionally, the effect of different doses of statin may also vary. Rutter et al. (26) conducted a double-blind clinical study on the therapeutic effect of different doses of atorvastatin on DN. It followed 60 patients with DN treated with atorvastatin $80 \mathrm{mg} /$ day and 59 patients treated with atorvastatin $10 \mathrm{mg} /$ day for 2 years and found that there were no statistically significant differences in renal function between patients taking highdose or low-dose atorvastatin for more than 2 years.

There were other indicators to assess the effect of statins, such as urinary 8-hydroxydeoxyguanosine (8-OHdG) and the liver-type fatty acid-binding protein (L-FABP), Urinary $8-\mathrm{OHdG}$ is a sensitive biomarker of oxidative DNA damage caused by DN, and L-FABP plays a key role in fatty acid metabolism in proximal tubules (27). We didn't identify enough studies to start a analysis, but only in study (8), it was reported that statins (rosuvastatin) could significantly decrease the 8-OHdG and L-FABP level.

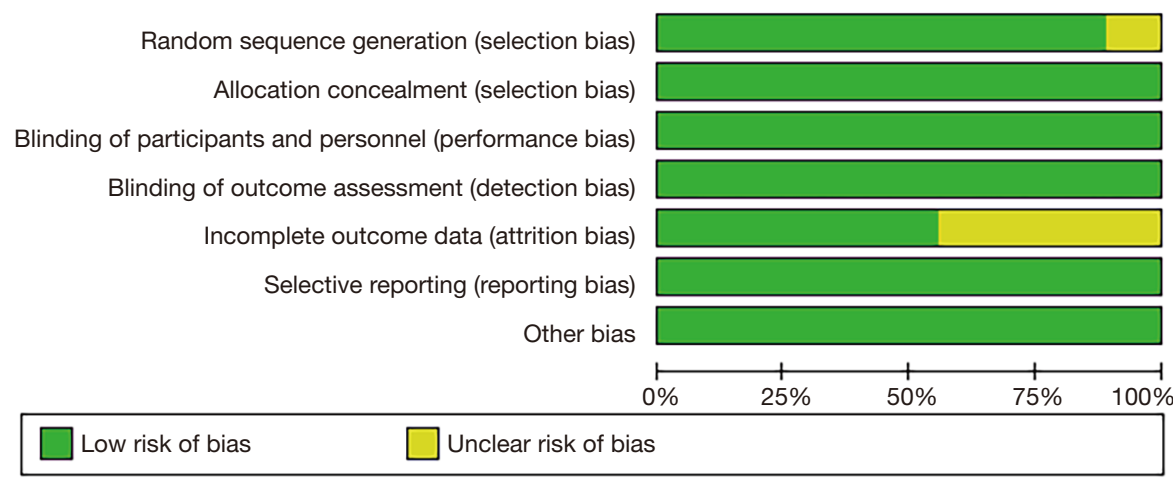

Figure 3 Risk of bias assessment chart of included articles. 


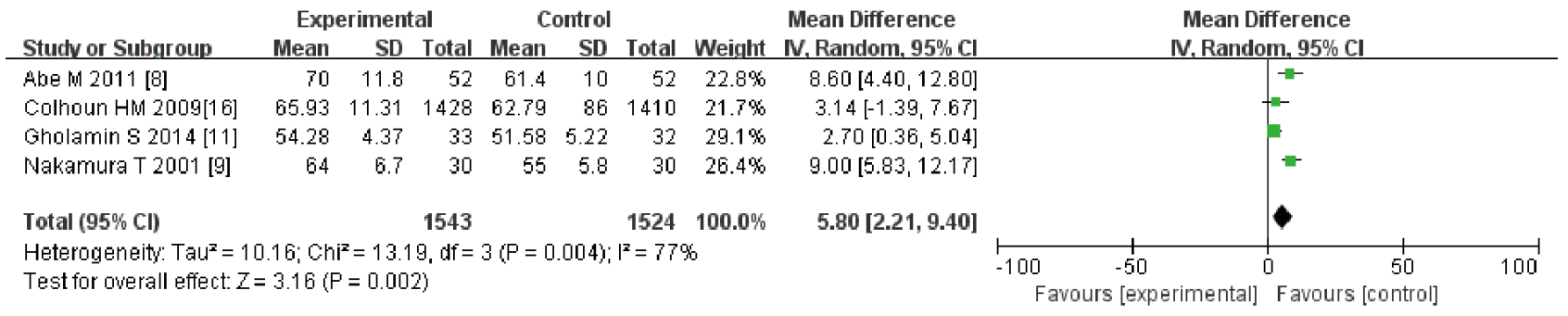

Figure 4 Effect of statins on eGFR in patients $(8,9,11,16)$. eGFR, estimated glomerular filtration rate.

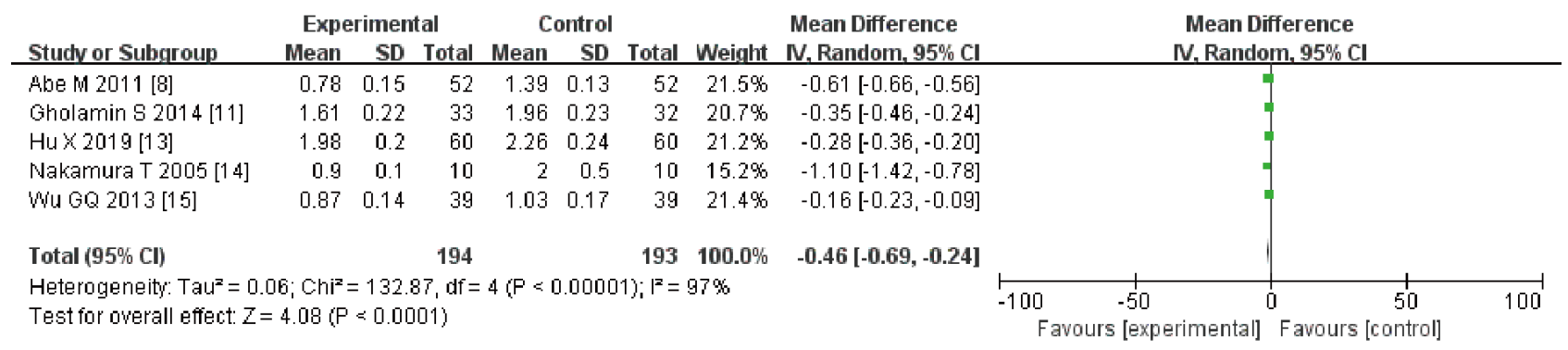

Figure 5 Effect of statins on SCR in patients (8,11,13-15). SCR, serum creatinine.

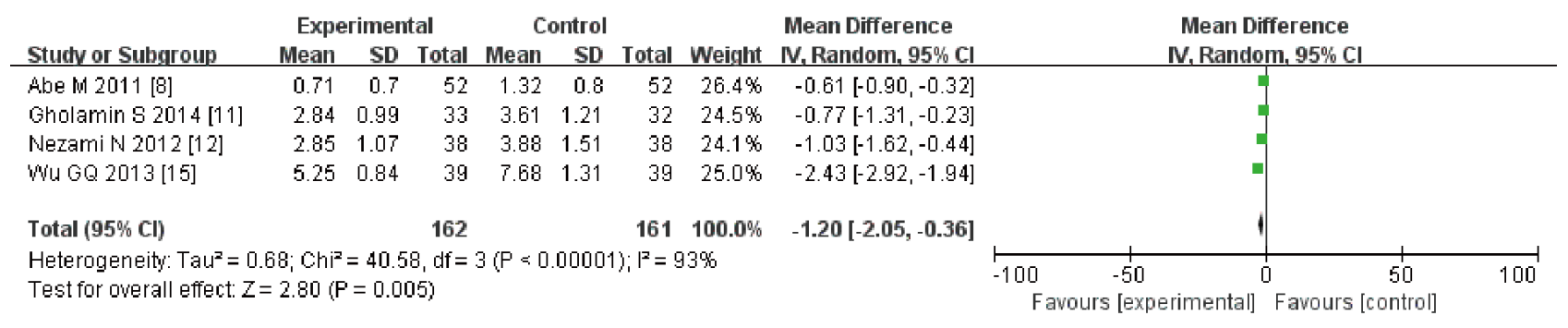

Figure 6 Effect of statins on serum hs-CRP in patients $(8,11,12,15)$. hs-CRP, high-sensitivity C-reactive protein.

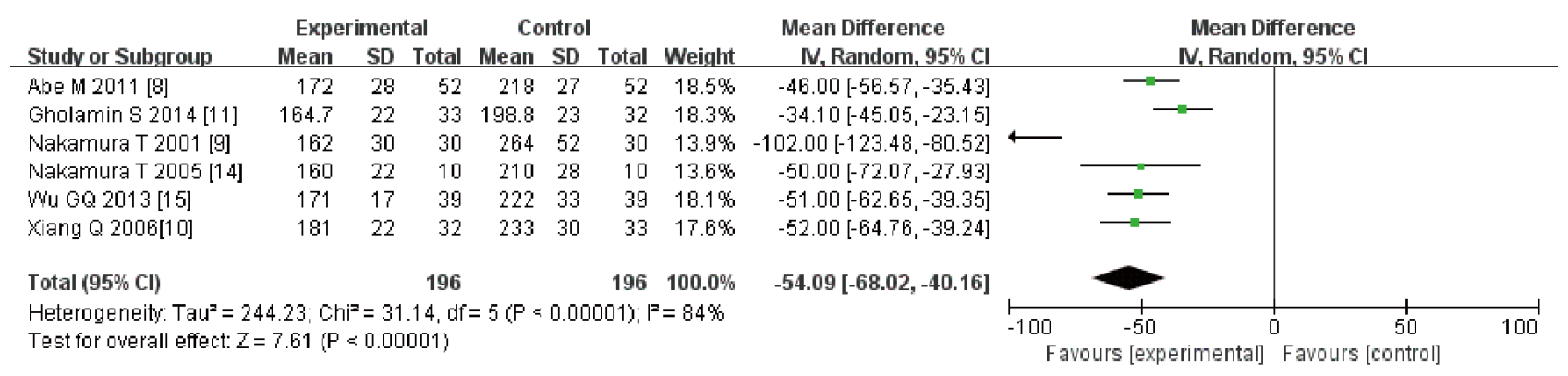

Figure 7 Effect of statins on serum TC in patients $(8-11,14,15)$. TC, total cholesterol. 


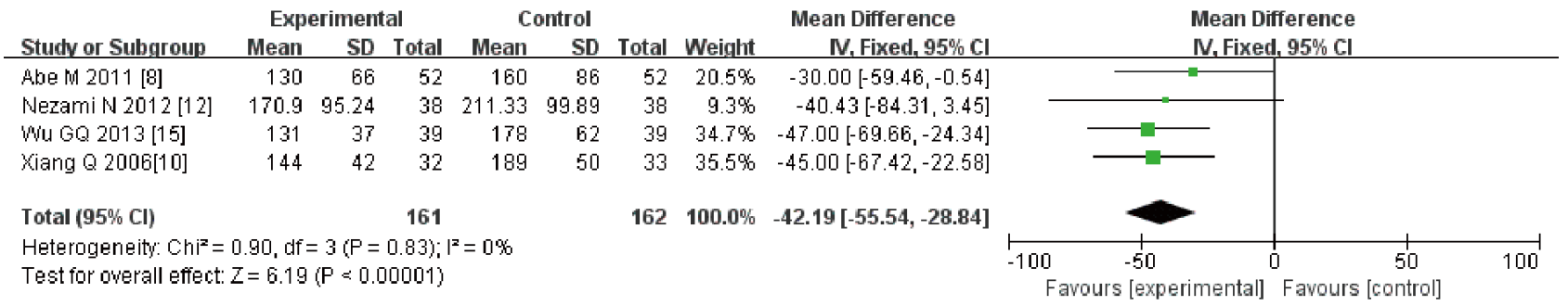

Figure 8 Effect of statins on serum TG in patients $(8,10,12,15)$. TG, total triglyceride.

Hematoxylin eosin staining (HE) can observe the pathological changes of DN, but we didn't identify any studies to report the results (28).

Adverse reactions to statins were not mentioned in the articles included in this study, and some studies (29) concluded that statins do not appear to reduce the risk of esophageal variceal bleeding and spontaneous bacterial peritonitis. A 5-year follow-up study by Baigent et al. (30) showed that statin therapy had no significant side effects, which greatly reduced the incidence of cardiovascular events and stroke.

In this study, only eight articles were included. Although the quality of the articles was satisfactory, it can also cause depletion bias. Furthermore, the number of articles included was small, the sample size of participants was small, and a publication bias analysis could not be performed. Therefore, the therapeutic effect of statins on DN needs to be supported by evidence from higher quality RCT studies.

\section{Conclusions}

In this meta-analysis, nine articles were included. Metaanalysis showed that the application of statins in the treatment of DN could significantly increase eGFR, reduce SCR, reduce CRP level, and reduce blood lipid level, thus reducing the inflammatory response and protecting the kidney.

\section{Acknowledgments}

Funding: None.

\section{Footnote}

Reporting Checklist: The authors have completed the PRISMA reporting checklist. Available at https://dx.doi. org/10.21037/apm-21-2673

Conflicts of Interest: All authors have completed the ICMJE uniform disclosure form (available at https://dx.doi. org/10.21037/apm-21-2673). The authors have no conflicts of interest to declare.

Ethical Statement: The authors are accountable for all aspects of the work in ensuring that questions related to the accuracy or integrity of any part of the work are appropriately investigated and resolved.

Open Access Statement: This is an Open Access article distributed in accordance with the Creative Commons Attribution-NonCommercial-NoDerivs 4.0 International License (CC BY-NC-ND 4.0), which permits the noncommercial replication and distribution of the article with the strict proviso that no changes or edits are made and the original work is properly cited (including links to both the formal publication through the relevant DOI and the license). See: https://creativecommons.org/licenses/by-nc-nd/4.0/.

\section{References}

1. Ding T, Wang S, Zhang X, et al. Kidney protection effects of dihydroquercetin on diabetic nephropathy through suppressing ROS and NLRP3 inflammasome. Phytomedicine 2018;41:45-53.

2. Sheng X, Dong Y, Cheng D, et al. Efficacy and safety of Bailing capsules in the treat-ment of type 2 diabetic nephropathy: a meta-analysis. Ann Palliat Med 2020;9:3885-98.

3. Radcliffe NJ, Seah JM, Clarke M, et al. Clinical predictive factors in diabetic kidney disease progression. J Diabetes Investig 2017;8:6-18.

4. Karatas A, Turkmen E, Erdem E, et al. Monocyte to high-density lipoprotein cholesterol ratio in patients with 
diabetes mellitus and diabetic nephropathy. Biomark Med 2018;12:953-9.

5. Newman CB, Preiss D, Tobert JA, et al. Statin Safety and Associated Adverse Events: A Scientific Statement From the American Heart Association. Arterioscler Thromb Vasc Biol 2019;39:e38-81.

6. Shen $X$, Zhang Z, Zhang X, et al. Efficacy of statins in patients with diabetic nephropathy: a meta-analysis of randomized controlled trials. Lipids Health Dis 2016;15:179.

7. Makaryus AN, McFarlane SI. Diabetes insipidus: diagnosis and treatment of a complex disease. Cleve Clin J Med 2006; 73:65-71.

8. Abe M, Maruyama N, Okada K, et al. Effects of lipidlowering therapy with rosuvastatin on kidney function and oxidative stress in patients with diabetic nephropathy. $\mathrm{J}$ Atheroscler Thromb 2011;18:1018-28.

9. Nakamura T, Ushiyama C, Hirokawa K, et al. Effect of cerivastatin on urinary albumin excretion and plasma endothelin- 1 concentrations in type 2 diabetes patients with microalbuminuria and dyslipidemia. Am J Nephrol 2001;21:449-54.

10. Xiang Q, Yin YS, Li XL, et al. The clinical observation of Simvastatin and irbesartan used or incombination alone for treating early diabetic nephropathy. Chinese Journal of Modern Applied Pharmacy 2006;(S2):823-5.

11. Gholamin S, Razavi SM, Taghavi-Garmestani SM, et al. Lovastatin for reduction of leptin in nondialysis patients with type 2 diabetic nephropathy. Iran J Kidney Dis 2014;8:201-6.

12. Nezami N, Safa J, Salari B, et al. Effect of lovastatin therapy and withdrawal on serum uric acid level in people with type 2 diabetic nephropathy. Nucleosides Nucleotides Nucleic Acids 2012;31:353-63.

13. Hu X, Li HY, Song B, et al. Effect of atorvastatin on renal fibrosis, oxidative stress and related factors in patients with diabetic nephropathy. Journal of Hainan Medical University 2019;25:662-5.

14. Nakamura T, Sugaya T, Kawagoe Y, et al. Effect of pitavastatin on urinary liver-type fatty acid-binding protein levels in patients with early diabetic nephropathy. Diabetes Care 2005;28:2728-32.

15. Wu GQ, Gao X, Mei CL. Clinical Observe Effect of Atorvastatin Combined with Benazepril Treatment of Early Diabetic Nephropathy. Chinese Journal of Integrated Traditional and Western Nephrology 2013;14:117-9.

16. Colhoun HM, Betteridge DJ, Durrington PN, et al. Effects of atorvastatin on kidney outcomes and cardiovascular disease in patients with diabetes: an analysis from the Collaborative Atorvastatin Diabetes Study (CARDS). Am J Kidney Dis 2009;54:810-9.

17. Qin X, Dong H, Fang K, et al. The effect of statins on renal outcomes in patients with diabetic kidney disease: A systematic review and meta-analysis. Diabetes Metab Res Rev 2017.

18. Cholesterol Treatment Trialists' (CTT) Collaboration; Fulcher J, O'Connell R, et al. Efficacy and safety of LDLlowering therapy among men and women: meta-analysis of individual data from 174,000 participants in 27 randomised trials. Lancet 2015;385:1397-405.

19. Zhang L, Zhang S, Yu Y, et al. Efficacy and safety of rosuvastatin vs. atorvastatin in lowering LDL cholesterol: A meta-analysis of trials with East Asian populations. Herz 2020;45:594-602.

20. Takazakura A, Sakurai M, Bando Y, et al. Renoprotective effects of atorvastatin compared with pravastatin on progression of early diabetic nephropathy. J Diabetes Investig 2015;6:346-53.

21. Bonaterra GA, Bender K, Wilhelm B, et al. Effect of cholesterol re-supplementation and atorvastatin on plaque composition in the thoracic aorta of New Zealand white rabbits. BMC Cardiovasc Disord 2020;20:420.

22. van der Ploeg MA, Floriani C, Achterberg WP, et al. Recommendations for (Discontinuation of) Statin Treatment in Older Adults: Review of Guidelines. J Am Geriatr Soc 2020;68:417-25.

23. Vlad A, Vlad M, Petrica L, et al. Therapy with atorvastatin versus rosuvastatin reduces urinary podocytes, podocyteassociated molecules, and proximal tubule dysfunction biomarkers in patients with type 2 diabetes mellitus: a pilot study. Ren Fail 2017;39:112-9.

24. Kimura S, Inoguchi T, Yokomizo H, et al. Randomized comparison of pitavastatin and pravastatin treatment on the reduction of urinary albumin in patients with type 2 diabetic nephropathy. Diabetes Obes Metab 2012;14:666-9.

25. de Zeeuw D, Anzalone DA, Cain VA, et al. Renal effects of atorvastatin and rosuvastatin in patients with diabetes who have progressive renal disease (PLANET I): a randomised clinical trial. Lancet Diabetes Endocrinol 2015;3:181-90.

26. Rutter MK, Prais HR, Charlton-Menys V, et al. Protection Against Nephropathy in Diabetes with Atorvastatin (PANDA): a randomized double-blind placebo-controlled trial of high- vs. low-dose atorvastatin(1). Diabet Med 2011;28:100-8. 
27. Kanauchi M, Nishioka H, Hashimoto T. Oxidative DNA damage and tubulointerstitial injury in diabetic nephropathy. Nephron 2002;91:327-9.

28. Huang $\mathrm{H}, \mathrm{Ni} \mathrm{H}, \mathrm{Ma} \mathrm{K}$, et al. ANGPTL2 regulates autophagy through the MEK/ERK/Nrf-1 pathway and affects the progression of renal fibrosis in diabetic nephropathy. Am J Transl Res 2019;11:5472-86.

29. Gu Y, Yang X, Liang H, et al. Comprehensive evaluation of effects and safety of statin on the progression of liver

Cite this article as: $\mathrm{Lv} \mathrm{J}$, Ren C, Hu Q. Effect of statins on the treatment of early diabetic nephropathy: a systematic review and meta-analysis of nine randomized controlled trials. Ann Palliat Med 2021;10(11):11548-11557. doi: 10.21037/apm-21-2673 cirrhosis: a systematic review and meta-analysis. BMC Gastroenterol 2019;19:231.

30. Baigent C, Keech A, Kearney PM, et al. Efficacy and safety of cholesterol-lowering treatment: prospective metaanalysis of data from 90,056 participants in 14 randomised trials of statins. Lancet 2005;366:1267-78.

(English Language Editor: J. Chapnick) 